
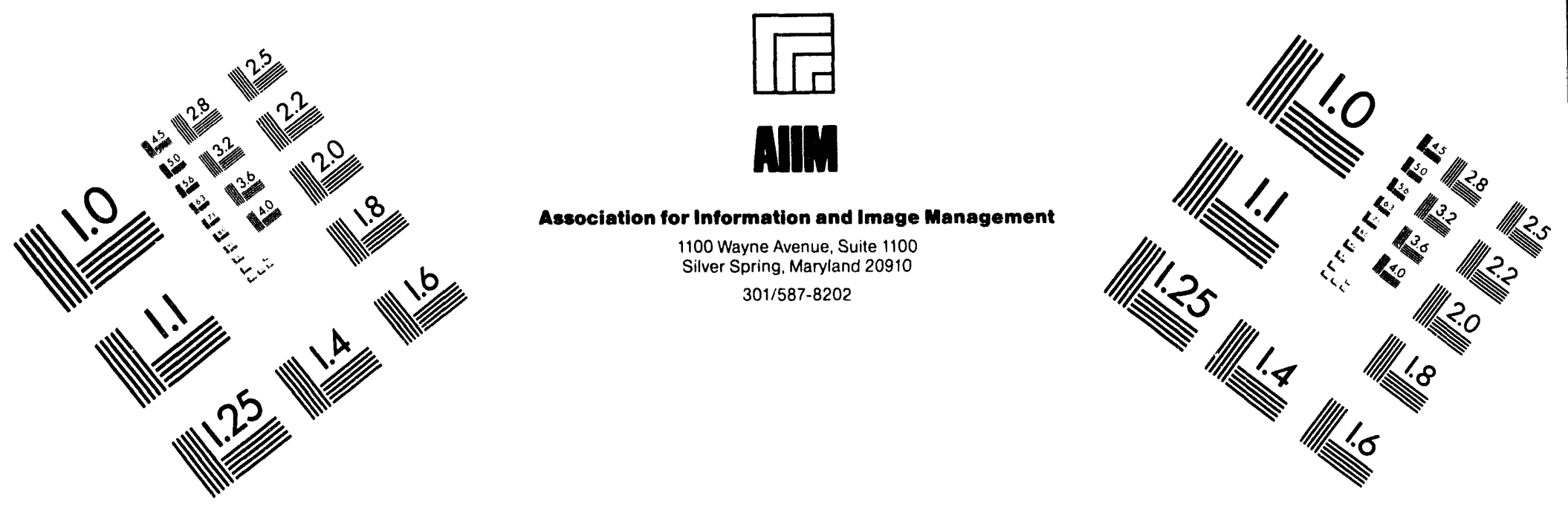

\title{
Centimeter
}

1m Inches
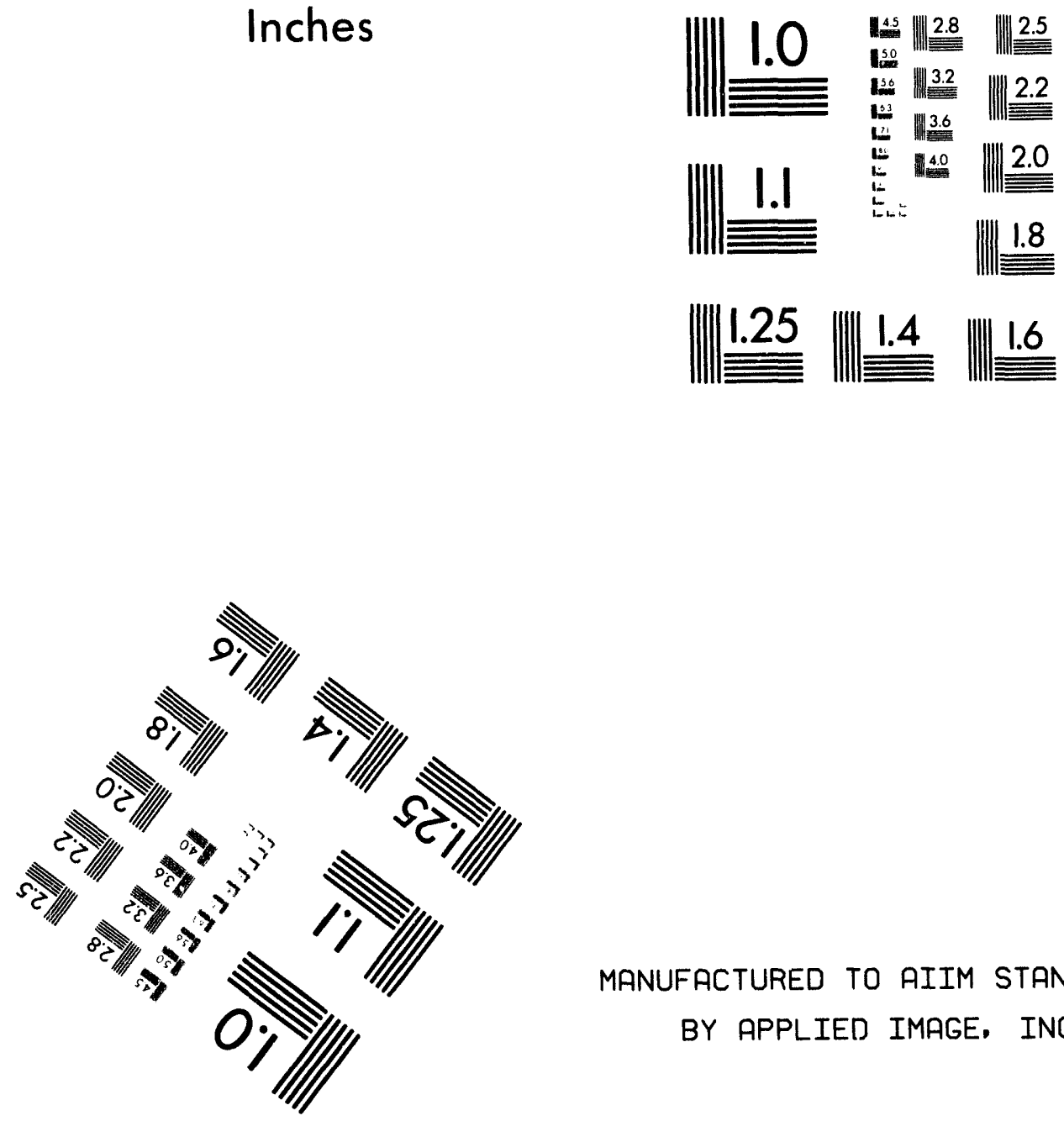

MANUFACTURED TO AIIM STANDARDS

BY APPLIED IMAGE, INC.

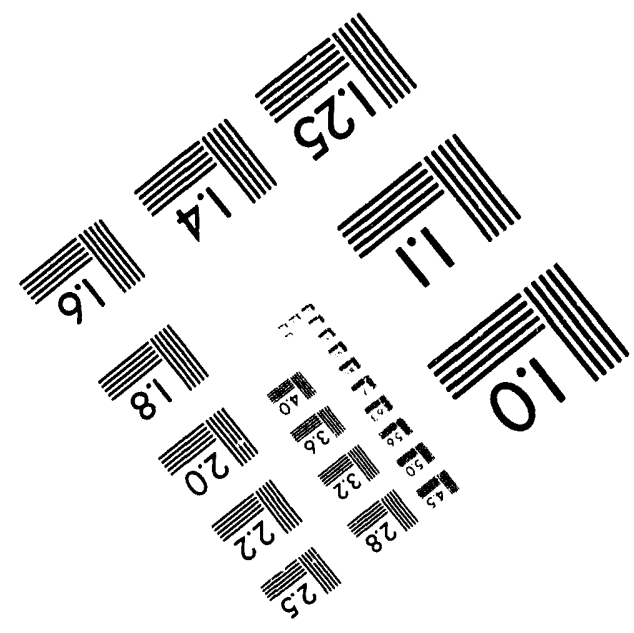



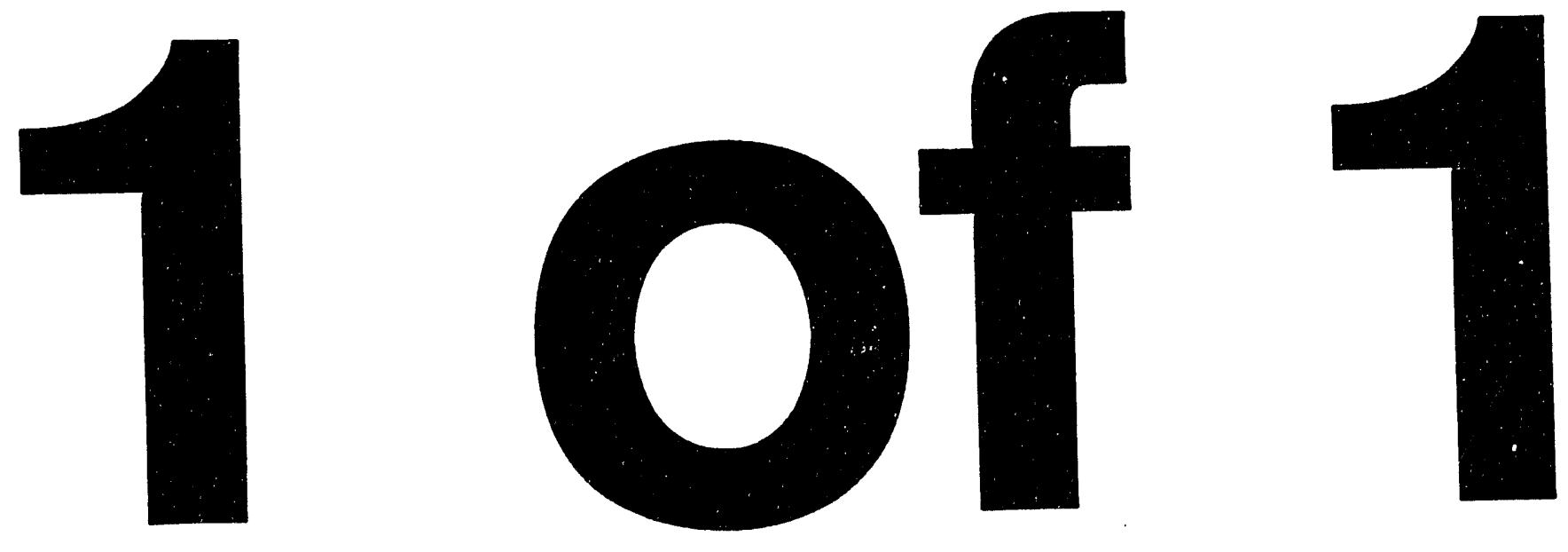


\title{
Conf- $940353--8$
}

\section{INVESTIGATING THE RESOLUTION OF RESISTIVITY ARRAYS USING INVERSE THEORY}

\author{
L.P. Beard \\ Environmental Sciences Division, Oak Ridge National Laboratory* , P.O. Box 2008, \\ Oak Ridge,Tennessee 37831-6317 \\ A.C. Tripp \\ Department of Geology and Geophysics, University of Utah, Salt Lake City, UT 84112
}

\begin{abstract}
We used a fast 2-D minimum structure inverse solution to examine the ability of different resistivity arrays to resolve structures given noisy synthetic data. We found that the resistivity models obtained from inversion of the dipole-dipole data were usually very similar to the estimated models from pole-dipole data, and both were superior to estimated models from pole-pole data. This is because noisy dipole-dipole or pole-dipole data define geoelectric structures much more precisely than an equivalent set of noisy pole-pole data. One structure where the dipole-dipole array appeared decidedly superior was the prism beneath a conductive overburden. The final estimated models from the pole-pole data were more poorly resolved than the final models from the other arrays. Our results show that the pole-pole data is a poor choice if vertical resolution of a geoelectric structure is crucial. Furthermore, if pole-pole data contains even a fraction of a percent of noise, the transformation of such data through superposition to equivalent data of other array types is virtually impossible, and significant information is lost.
\end{abstract}

\section{INTRODUCTION}

In planning a resistivity survey, it would be useful to have some idea as to what type of an electrode array is best suited for searching for a particular type of buried structure. One way to obtain this information would be to simulate resistivity data for the structure in question, and then invert the data for the subsurface resistivity distribution. In a previous paper, we described in detail a fast approximate inversion algorithm for dc resistivity data expressly designed for situations in which subsurface resistivity variations are gradual and resistivity contrasts low (Beard et al., in review) and successfully applied this inversion algorithm to both noisy synthetic and real dipole-dipole array data. In this paper, we examine inverse solutions obtained from noisy synthetic resistivity data computed using three common array configurations: the dipole-dipole, pole-dipole, and pole-pole arrays (Figure 1). Two targets, shown in Figure 2, are considered: (1) two conductive bodies in close proximity to one another, and (2) a conductor buried beneath a conductive surface unit. These targets were chosen because they represent common targets in geophysical exploration and because each of these models illustrate limitations inherent to resistivity methods.

Our study is unique in that the different arrays are compared on the basis of 2-D inversion results. The estimated models we obtained from our 2-D inversions of noisy synthetic data show

The submitted manuscript has been authored by a contractor of the U.S. Government under contract No. DE-AC05-84OR21400. Accordingly, the U.S. Government retains a non-exclusive, royalty-free license to publish or reproduce the published form of this contribution, or allow others to do so, for U.S. Government purposes.

DISTRIBUTION OF THIS DOCUMENT IS UNLIMITIE

* Managed by Martin Marietta Energy Systems, Inc. under contract No. DE-AC05-84OR21400 for with the U.S. Department of Energy. 
the resolution of each array. This study also indicates which types of structures are best resolved by these arrays, and shows the problems one might expect to encounter when trying to image certain structures with inversion techniques. Though a number of studies comparing arrays are in the literature, none have used this approach because previously existing inversion algorithms have either been written for a specific array type, or the inversions were relatively slow, requiring several hours to complete an iterative solution, thus making a comparative study laborious. The iterative, 2-D low-contrast inversion algorithm, requiring only a few minutes of CPU time on a modest workstation, enabled us to invert data for different array types quickly and efficiently. We were, of necessity, limited to models having relatively low resistivity contrasts. However, the general conclusions we reach in this study are most likely applicable to high contrasts as well since the dc resistivity response for most common arrays saturates at contrasts of less than 100:1 at modest noise levels.

Several studies have been performed which compare different array types. Coggon (1973) used finite element forward modeling to examine the dipole-dipole, pole-dipole, and gradient array responses over several different geologic structures. He concluded that the dipole-dipole and poledipole arrays yielded the largest anomalies, but that the dipole-dipole array was insensitive to the dip of a conductor and the pole-dipole's asymmetric response sometimes made interpretation difficult. Ward (1990) provides an excellent review of different resistivity arrays and their responses.

A number of authors have studied the "depth of investigation" of different arrays. Roy and Apparao (1971) examined several arrays and concluded that while the depth of investigation of the pole-pole array is the greatest of the several arrays they examined, it also possesses the least vertical resolution. Conversely, the Wenner, Schlumberger, and dipole-dipole arrays had small depths of investigation, but also had high vertical resolutions. Brizzolari and Bernabini (1979) compared Schlumberger array profiles with various focused arrays and concluded that focused arrays hold an advantage over more common array types in discriminating certain structures, such as buried cavities or tunnels. Beard and Morgan (1991) compared Schlumberger and Wenner arrays by applying layered earth inversions to synthetic resistivity sounding data from 2-D structures. They concluded that, in most cases, 1-D layered earth solutions "stitched together" from the Schlumberger array led to less ambiguous interpretations than did similar Wenner solutions.

\section{METHODOLOGY}

For the structures in Figure 2, we generated synthetic data using modified versions of GRIDIP, a 3-D dc resistivity/IP forward modeling program which uses the volume integral equation method (Beasley and Ward, 1986). For each structure, we generated dipole-dipole, poledipole, and pole-pole data. These data were contaminated with 5\% Gaussian noise, then presented in pseudosection form. For these arrays, this level of noise is moderate and may be routinely attained. Otherwise, these data sets were kept "equivalent" in the sense that the data lines for each array type began at the same location and were the same length. Each line ran over the center of the body perpendicular to strike and the number of $\mathrm{N}$-spacings were $\mathrm{N}=1-6$ for the dipole-dipole and pole-dipole arrays and $\mathrm{N}=1-7$ for the pole-pole. The extra pole-pole spacing kept the total line length the same for an equivalent number of transmitter positions. The receiver a-spacing was the same for the pole-dipole and dipole-dipole arrays and the shortest distance between receiver and transmitter electrodes, the $\mathrm{N}=1$ spacing, was the same for all arrays. The pole-pole and poledipole data were computed with the "infinite" current electrode 40 dipole lengths to the left of the origin. Each data set was inverted using the 2-D low-contrast, minimum structure inverse solution described in Beard et al. (in review). In all cases, the inversions began with an initial guess equal to the exact background resistivity of $100 \mathrm{ohm}-\mathrm{m}$. All other parameters involved in the inversions were held the same at the start of each inversion: the size of the discretization grid in $x, y$, and $z$ 
directions, the size of the discretization cells, the range over which the smoothing parameter $\mu$, used in the minimum structure inverse algorithm, was allowed to vary, the starting value of $\mu$, and the starting and ending points of the resistivity lines. The low-contrast inversion algorithm iterates on a 3-D grid of cells, but constrains the resistivities of the cells in any given row in the strike direction to be constant, thus making the inversion 2-D, but with end corrections in the strike direction. For each inversion, the strike length of the 3-D grid was set exactly equal to the strike length of the body from which the synthetic data were generated. Though not practical for actual field data, this condition ensured optimal conditions for the inversion. Had we chosen a longer discretization grid in the strike direction, the effect on the final inversion models would have been slight, and the comparisons shown below would still hold.

\section{MODEL COMPARISONS}

The results shown in the following inversions are scale independent, so all our length measurements are in terms of dipole lengths, that length being the receiver electrode separation in the case of the dipole-dipole or pole-dipole arrays. The pole-pole array electrode spacings are also normalized to dipole lengths. For example, the $\mathrm{N}=2$ pole-pole spacing corresponds to the transmitter and receiver pole being two dipole lengths apart. Dimensions of anomalous bodies are also expressed in dipole lengths, so that in the following section if a body is said to have dimensions $2 \times 6 \times 2$, this should be taken to mean two dipole lengths in the $x$ direction, six dipole lengths in the $y$ direction, and two dipole lengths in the $z$ direction.

\section{Two proximate, conductive bodies}

Environmental targets, such as buried waste trenches, make the ability to discriminate proximate, but separate, structures an important factor in choosing an appropriate array. To compare the abilities of the dipole-dipole, pole-dipole, and pole-pole arrays in laterally and vertically resolving two adjacent conductors, we computed synthetic data from the model shown in Figure 2a. Both bodies have resistivities of $25 \mathrm{ohm}-\mathrm{m}$ in a $100 \mathrm{ohm}-\mathrm{m}$ half space. The larger body on the left has dimensions $2 \times 6 \times 2$ and the smaller body on the right is $1 \times 6 \times 1$. They were separated by one dipole length. The depth to the top of each is 0.5 dipole lengths. Synthetic data, contaminated with 5\% Gaussian noise, are shown as pseudosections in Figure 3 a-c. The dipoledipole pseudosections, shown in Figure 3a, show ambiguous evidence of two separate bodies. To interpret these data as two spatially separate bodies is difficult based on simple inspection. Interpretation by inspection of the pole-dipole data, shown in Figure $3 b$, is even more difficult. The asymmetry of these data compounds the problem of interpretation by inspection. The polepole array, with its poor resolution, yields an apparent resistivity pseudosection, shown in Figure $3 \mathrm{c}$, which hints at a shallow conductive structure, but gives no evidence that there are two separate bodies.

Proceeding from an initial guess equal to the exact background resistivity, minimum structure inversion of the dipole-dipole data images two separate bodies, as shown in Figure 4a. The smaller body is less well resolved than the larger body, both in terms of resistivity. The top two layers of cells in the larger body are accurately estimated at $10-30 \mathrm{ohm}-\mathrm{m}$. The resistivity of the smaller body is slightly overestimated.

The final estimated models of the pole-dipole inversions are shown in Figure $4 \mathrm{~b}$. The image from the resistivity inversion is very similar to that of the inversion of the dipole-dipole data. The bodies appear separate, though the resistivities of the cells between the bodies fall in the 45-65 ohm-m range, whereas the dipole-dipole inversion yielded higher resistivities in this same location, making the separation between the bodies appear more distinct. 
Given the ambiguous nature of the pole-pole resistivity data, the final estimated resistivity model from pole-pole resistivity inversion, shown in Figure 4c, can hardly be expected to resolve two separate bodies. What appears in the resistivity inversion is a broad resistivity low encompassing both bodies, but with the resistivities of the cells of the larger body being even lower. The resistivity estimates of the upper layers of cells in the larger body are in the 20-45 ohm-m range, and 45-65 ohm-m for the smaller body. The resistivities of the cells between the bodies range from $30 \mathrm{ohm}-\mathrm{m}$ to $65 \mathrm{ohm}-\mathrm{m}$, obscuring the smaller body.

\section{Conductor masked by conductive overburden}

A structure that would ordinarily have a large electrical response associated with it may have the magnitude of the response weakened by the presence of a conductive layer at the surface. The surface layer channels the electric current from the surface electrodes, allowing only a fraction of the current to interact with the target of interest. Thick conductive overburden is frequently encountered in arid regions such as parts of Alsstralia or the western United States, and in the areas of glacial till in the midwestern and northeastern United States. One way to avoid this problem is to use cross-borehole arrays in which the transmitters and receivers are below the conductive layer. If this is not an option, the geophysicist must somehow attempt to incorporate the response of the near-surface in the interpretation. The following example illustrates the degree to which data from different arrays is affected by conductive overburden.

We computed synthetic data, adding 5\% Gaussian noise, for the model shown in Figure $2 \mathrm{~b}$. Our model consists of a $30 \mathrm{ohm}-\mathrm{m}$ conductor buried beneath a $20 \mathrm{ohm}-\mathrm{m}$ conductive layer with a background resistivity of $100 \mathrm{ohm}-\mathrm{m}$. The top of the body is 0.5 dipole lengths beneath the surface, and its dimensions are $2 \times 6 \times 2$. The conductive surface unit has dimensions $5 \times 6 \times 0.5$, and is centered over the top of the deeper body.

The apparent resistivity pseudosection corresponding to the different arrays are shown in Figure 5 a-c. The difference in the dipole-dipole and pole-dipole data is striking. The dipoledipole resistivity pseudosection is relatively symmetric, and shows evidence of a conductive body beneath the surface layer. The pole-dipole pseudosection shows a pronounced asymmetry. The resistivities are lower toward the left side of the pseudosection, i.e. in the direction of the transmitter pole at "infinity", located 40 dipole lengths to the left of the origin. Until the geophysicist gains considerable experience with pole-dipole data, such asymmetry can make interpretation by inspection difficult. In Figure 5c, we see the magnitude of the resistivity anomaly for the pole-pole array is considerably reduced in comparison with those of the other two arrays.

The final estimated models from the inversions are shown in Figure 6 a-c. For comparative purposes, we assumed exact knowledge of the thickness, resistivity and location of the nearsurface conductive unit. The surface unit was then modeled in an otherwise homogeneous halfspace of $100 \mathrm{ohm}-\mathrm{m}$, and its secondary response was computed. In the iterative inversion this response was then added to the secondary response of the deeper body. This approach ignores electrical interactions between the surface unit and the deeper body, but at relatively low resistivity contrasts, this interaction is small. Reasonable inverse models may be obtained with only rough estimates of the surface unit's resistivity, so long as the location of the body is fairly wellconstrained.

In the case of the final estimated model from the cipole-dipole inversion, shown in Figure 6a, the location of the conductive body is well-constrained, though the top of the body appears somewhat deeper than it actually is. This inaccuracy is partly due to our neglecting the small amount of interaction between the body. The dipole-dipole final estimated resistivity model is reasonable, with the resistivities of many cells falling in the $30-40 \mathrm{ohm}-\mathrm{m}$ range.

Final estimated models from the pole-dipole and pole-pole inversions are shown in Figures $6 \mathrm{~b}$ and $6 \mathrm{c}$. The image of the resistivity structure is less well-defined than the dipole-dipole images, but is otherwise similar. The weaker anomalies associated with the pole-pole data make accurate inverse estimates difficult. The body's resistivity, shown in Figure $6 \mathrm{c}$, is estimated to be at 
minimum 45 ohm-m, $50 \%$ higher than the true value of $30 \mathrm{ohm}-\mathrm{m}$, and the depth to the top of the low resistivity zone is estimated even deeper than with the dipole-dipole and pole-dipole inversions. Its lateral location is fairly well estimated.

In summary, if the conductive near-surface structure can be fairly well-constrained, inversions from data collected using the dipole-dipole array appear superior to pole-dipole or pole-pole inversions in imaging conductive structures beneath the near-surface layer. Inversion of pole-pole data was considerably less successful in imaging the deep structure. In this example, a relatively low contrast was used, both for the near-surface conductor and for the deeper body. If the nearsurface is made much more conductive or if the layer is thicker, the masking effect would be correspondingly greater.

\section{LIMITATIONS OF' POLE-POLE DATA}

Pole-pole resistivity data has recently undergone renewed popularity as electrical methods have been increasingly applied to environmental, engineering, and groundwater problems. Frangos (1992) showed how inverse theory can be applied to electric potentials to accurately locate leaks in lined waste ponds, the leak itself simulating one pole of the pole-pole array. Park and Van (1991) developed a 3-D inversion algorithm for pole-pole data. However, while the pole-pole array may occasionally hold logistical advantages over other electrode configurations, and while it may be very effective in specific applications, this study indicates it is inferior to other arrays as a geologic mapping tool. Its poor vertical depth resolution is its primary drawback. In theory, polepole data should, by use of superposition, be transformable to any other electrode arrangement. Indeed, some practitioners have lauded this as an advantage in collecting pole-pole data. Unfortunately, as we show below, even very minute levels of noise will prevent pole-pole data from being used in this flexible manner. It appears that once pole-pole data has been collected, the user is stuck with pole-pole data since additional information and resolution inherent in pole-dipole or dipole-dipole configurations is lost if noise levels exceed even a fraction of a percent.

In Figure 7a, we see a portion of a noise free pole-pole pseudosection computed from a line over the center of a $10 \mathrm{ohm}-\mathrm{m}$ cube of dimension $2 \times 2 \times 2$, buried 0.5 dipole length beneath the surface and centered at the origin. Figure $7 \mathrm{~b}$ shows a portion of a "perfect" noise free dipoledipole pseudosection computed from the same body. In Figure $7 \mathrm{c}$-e, we see dipole-dipole pseudosections computed from the pole-pole data after different levels of noise are added. At zero percent, the dipole-dipole data derived from differencing the pole-pole data are exactly the same as the "perfect" dipole-dipole data. A few percent change in the apparent resistivities occurs at even $0.1 \%$ noise, and considerably more at $0.4 \%$. At $1 \%$ noise, some dipole-dipole apparent resistivities obtained from differencing differ radically from the true apparent resistivities, and by $2 \%$ noise (not shown) some of the differenced dipole-dipole apparent resistivities become negative. It is thus clear that unless pole-pole data can be collected at exceedingly low noise levels on the order of $0.1 \%$, noisy pole-pole data cannot contain the same information as dipole-dipole data. While the pole-pole array may have many useful applications, it should not be considered a blanket array which covers all other electrode configurations.

\section{CONCLUSIONS AND RECOMMENDATIONS}

For mapping geoelectric structures using dc resistivity, the dipole-dipole array appears to hold only a slight advantage over the pole-dipole array in most situations. Both arrays were clearly superior to the pole-pole array in the cases presented. The main disadvantage of pole-dipole data is that it yields asymmetric psudosections over symmetric bodies. Accurate interpretation of poledipole pseudosections by inspection is difficult as the data may be misleading to inexperienced interpreters, making interpretation through forward modeling or inversion almost indispensible. In this study, the dipole-dipole best resolved a conductor masked by conductive overburden. The 
pole-pole array did a good job of resolving lateral resistivity variations. Conductive overburden affects dipole-dipole and pole-dipole arrays about equally.

Pole-pole data has poor vertical resolution, but may be reasonably effective in resolving lateral resistivity contrasts. It cannot be reliably transformed to other types of data unless noise levels can be kept at around $0.1 \%$ which, at present levels of technology, is a very difficult figure to attain.

Because our inversion algorithm is most accurate at low resistivity contrasts, all the examples in this study involved resistivity contrasts of 5:1 or less. We do not expect our general conclusions to be significantly altered for high resistivity contrasts, but a similar study using higher contrasts of 100:1 or more would be useful in ascertaining this.

\section{ACKNOWLEDGMENTS}

The authors thank the members of the University of Utah Consortium for Electromagnetic Modeling and Inversion (CEMI), whose collective support made this work possible: AtlasWireline, BHP-Utah Minerals, B.P. Research, CRA Exploration, FMC-Gold, Geothermal Energy Research and Development, Kennecott Exploration, Mobil Research and Development, Noranda, Schlumberger Research and Development, Shell Exploratie en Produktie Laboratorium, and Unocal.

\section{REFERENCES}

Beard, L.P., Hohmann, G.W., and Tripp, A.C., in review, Fast resistivity/IP inversion using a low-contrast approximation: Submitted to Geopl ysics.

Beard, L.P. and Morgan, F.D., 1991, Assessment of 2-D resistivity structures using 1-D inversion: Geophysics, 56, 874-883.

Beasley, C.W. and Ward, S.H., 1986, Three-dimensional mise-a-la-masse modeling applied to mapping fracture zones: Geophysics, 51, 98-113.

Brizzolari, E. and Bernabini, M., 1979, Comparison between Schlumberger electrode arrangement and some focused electrode arrangements in resistivity profiles: Geophys. Prosp., 27, 233-244.

Coggon, J.H., 1973, A comparison of IP electrode arrays: Geophysics, 38, 737-761.

Frangos, W., 1992, Electrical detection of leaks in lined waste disposal ponds: M.S. thesis, University of Utah, 49pp.

Hohmann, G.W., 1990, Three-dimensional IP models in Induced Polarization: Applications and Case Histories, Soc. Expl. Geophys. Investigations in Geophysics No. 4, 150-178.

Park, S.K. and Van, G.P., 1991, Inversion of pole-pole data for 3-D resistivity structure beneath arrays of electrodes: Geophysics, 56, 951-960.

Roy, A. and Apparao, A., 1971, Depth of investigation in direct current methods: Geophysics, 36, 943-959.

Ward, S.H., 1990, Resistivity and induced polarization methods in Geotechnical and Environmental Geophysics: Vol. 1, Soc. Expl. Geophys. Investigations in Geophysics No. 5, 147-189. 
(a)

dipole-dipole array

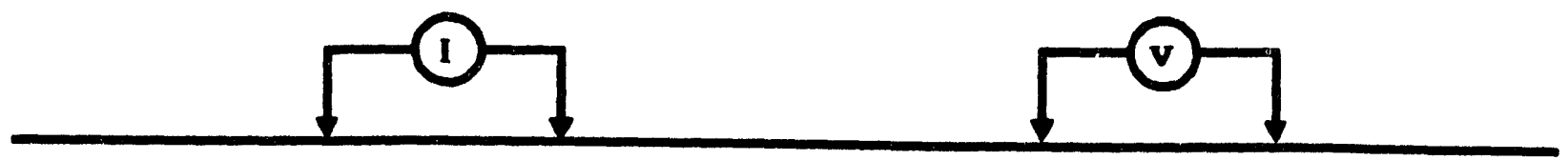

(b)

pole-dipole array

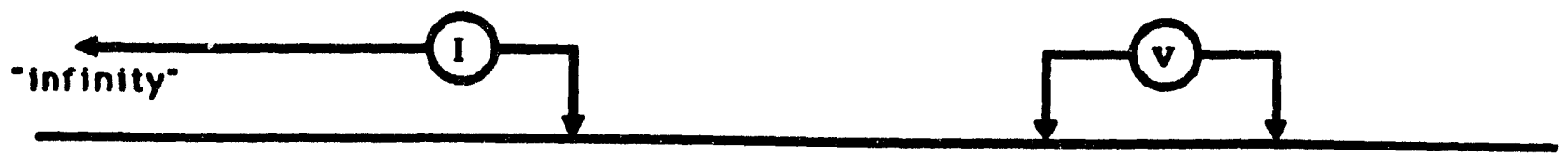

(c)

pole-pole array

(1)

-infinity"

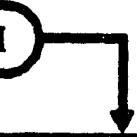

(v)

-infinity"

FIG. 1. Collinear resistivity arrays used in this study. (a) Dipole-dipole array. (b) Pole-dipole array. (c) Pole-pole array. 
two bodies dipole lengths

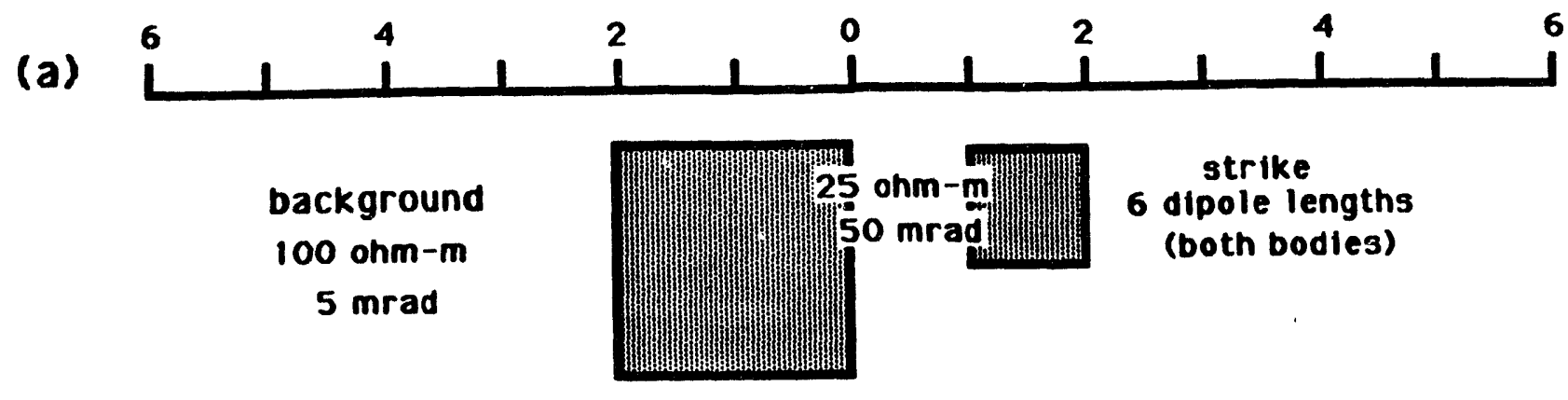

body beneath surface conductor dipole lengths

(b)

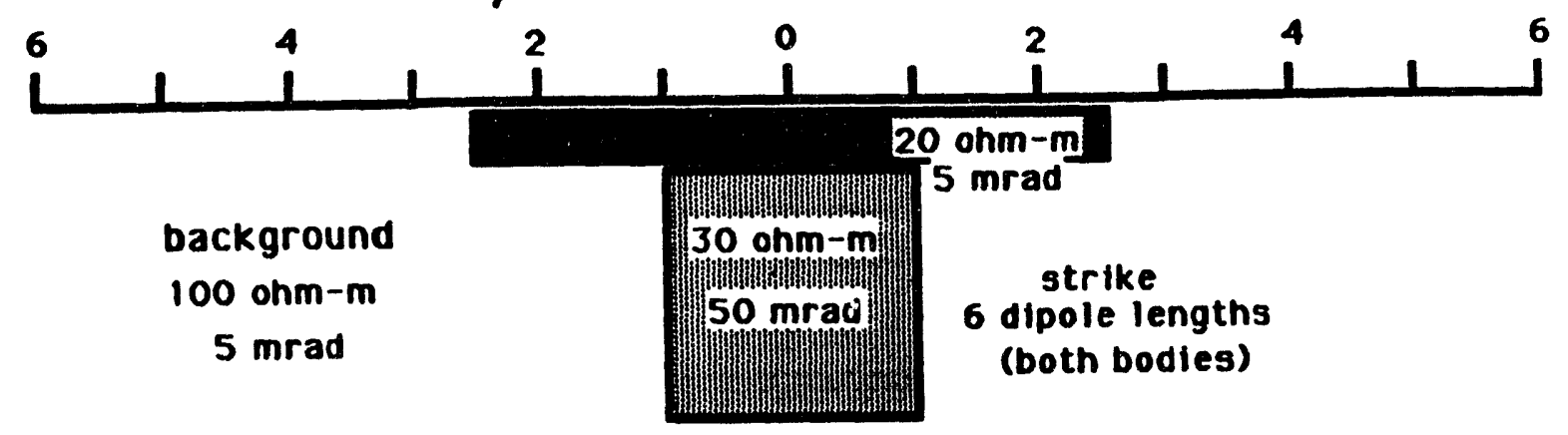

FIG. 2. Cross-sections of the models used to generate the synthetic apparent resistivity data. Strike lengths are shown in the figure. Data were simulated directly over the center of the bodies with the traverse perpendicular to the strike direction. Depth to the top of the bodies is one-half of a dipole length. (a) Two proximate conductive bodies. (b) Conductive body beneath a conductive near-surface unit. 
two bodies

(a)

dipole-dipole
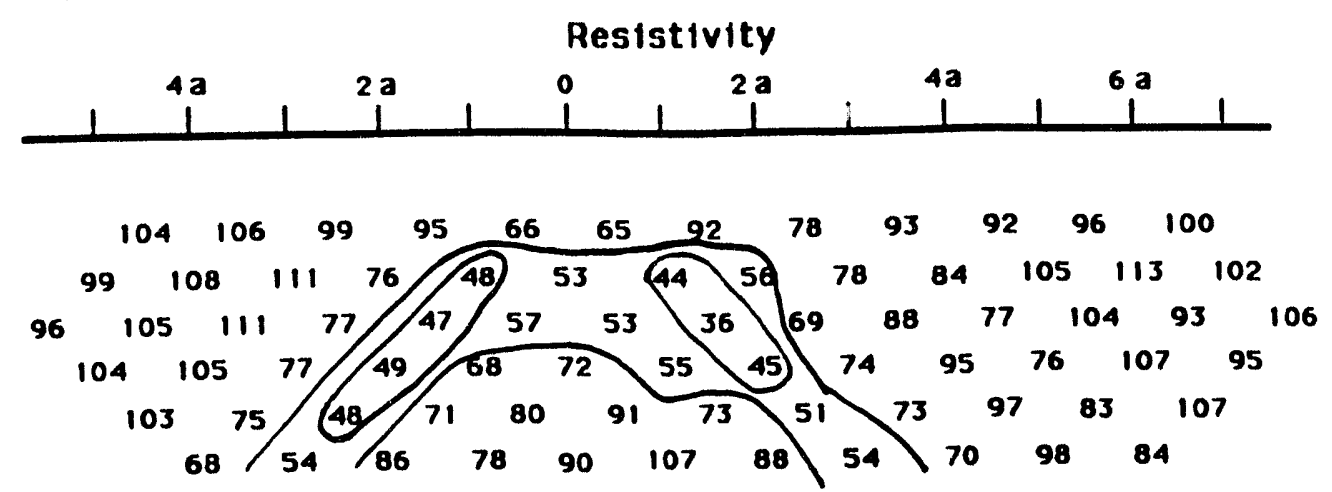

(b)

pole-dipole

\section{Resistivity}
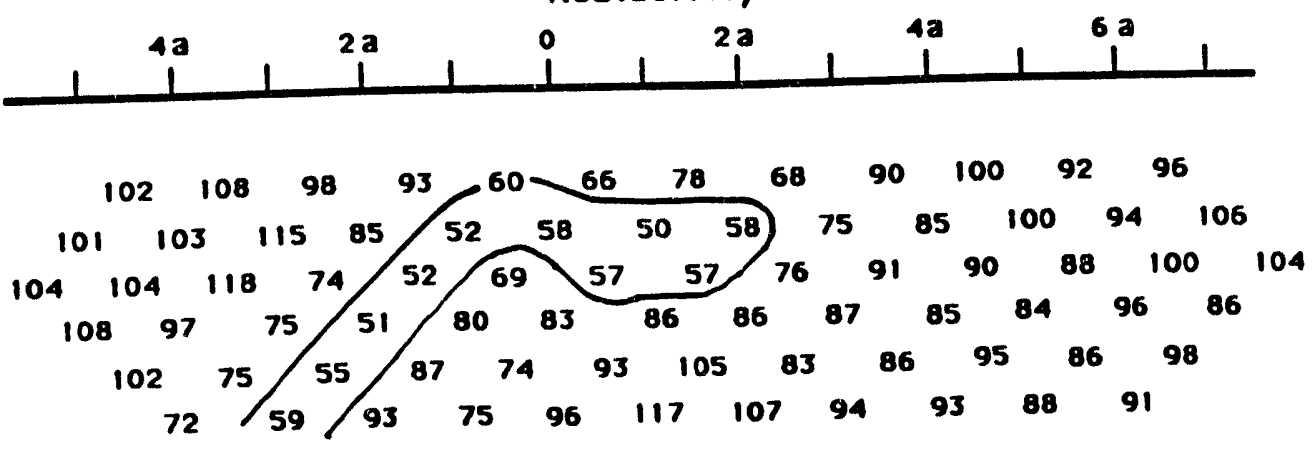

(c)

pole-pole

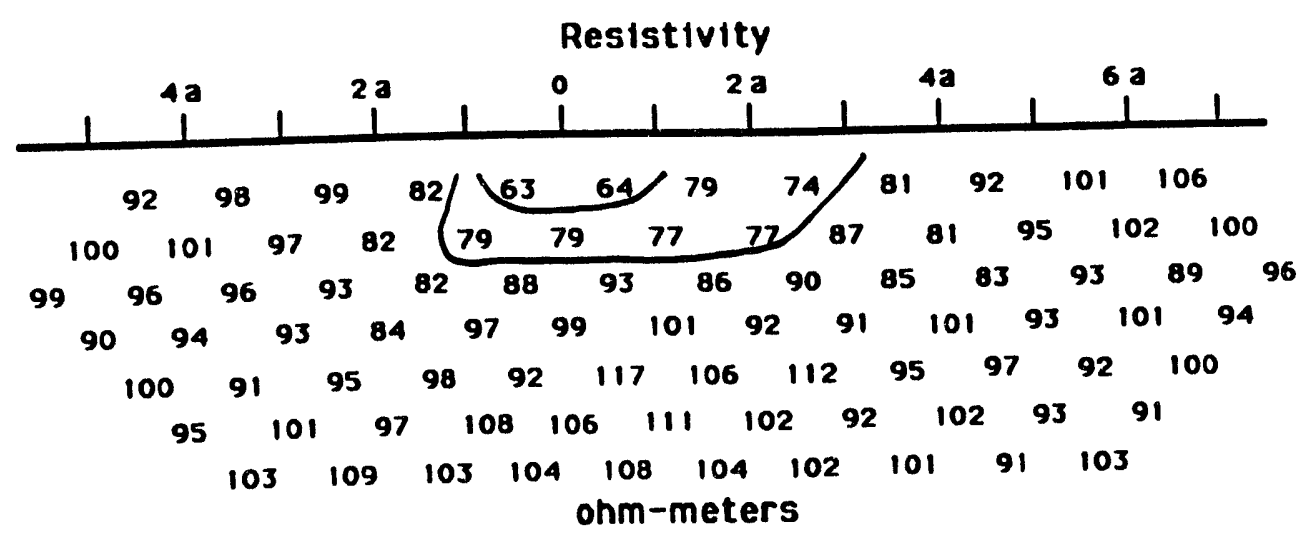

FIG. 3. Apparent resistivity with $5 \%$ Gaussian noise added for different arrays over two proximate conductors. (a) Dipole-dipole pseudosection. (b) Pole-dipole pseudosection. (c) Pole-pole pseudosection. 


$$
80^{\circ} I=z^{\wedge} \chi \text { Ki! }
$$

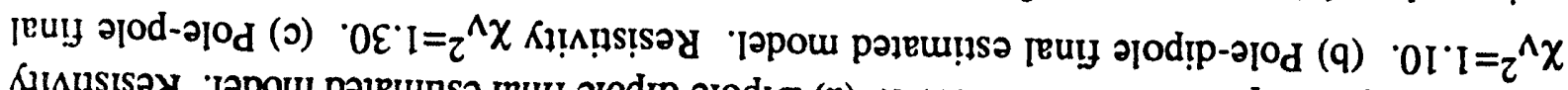

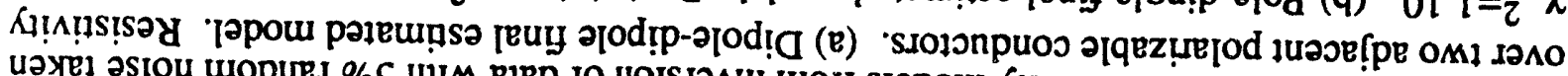

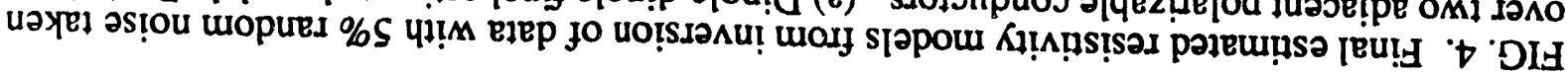
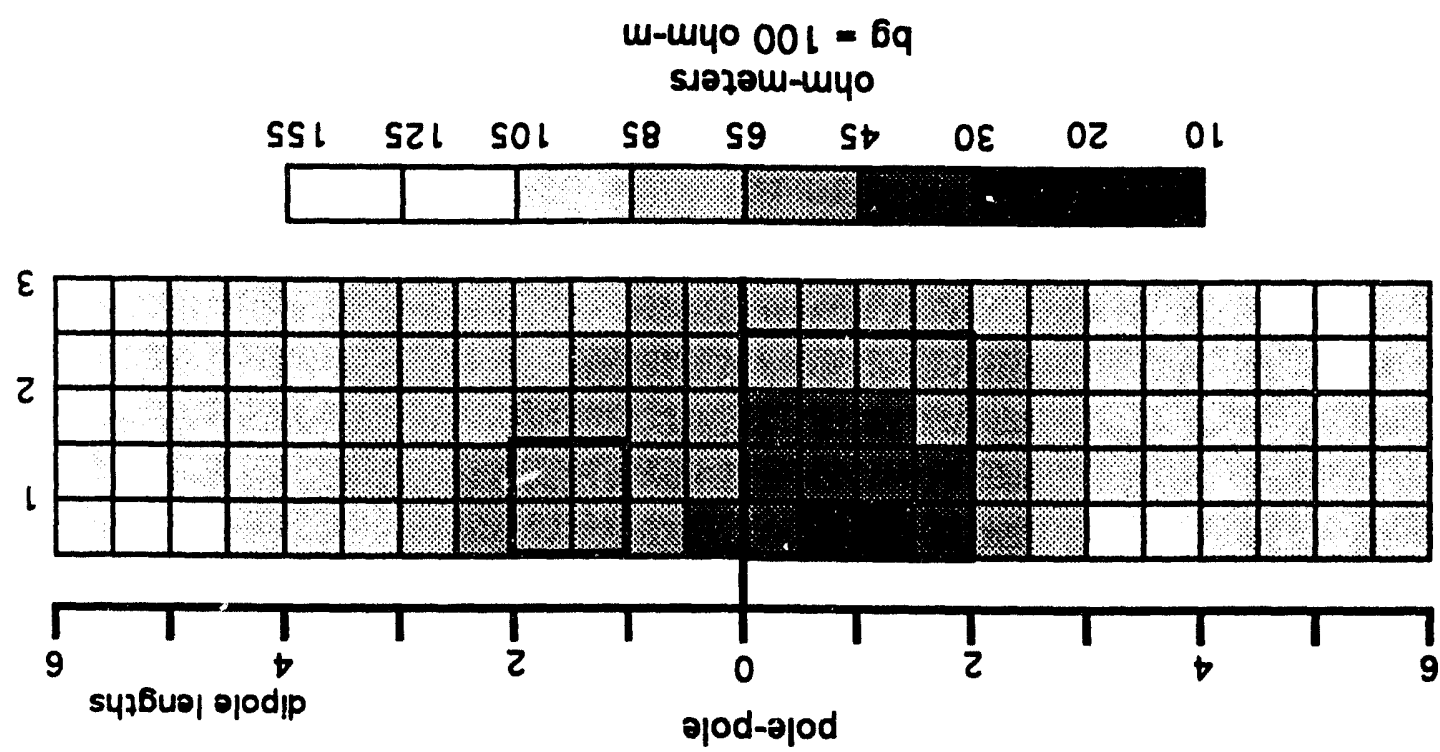

(ग)

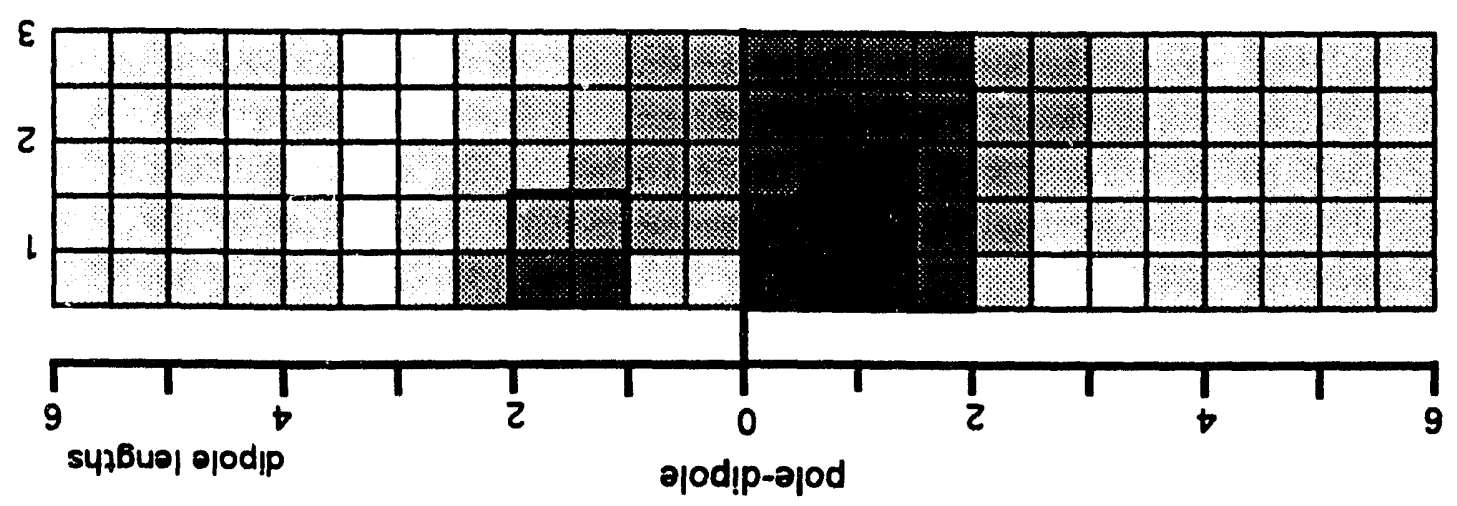

(q)

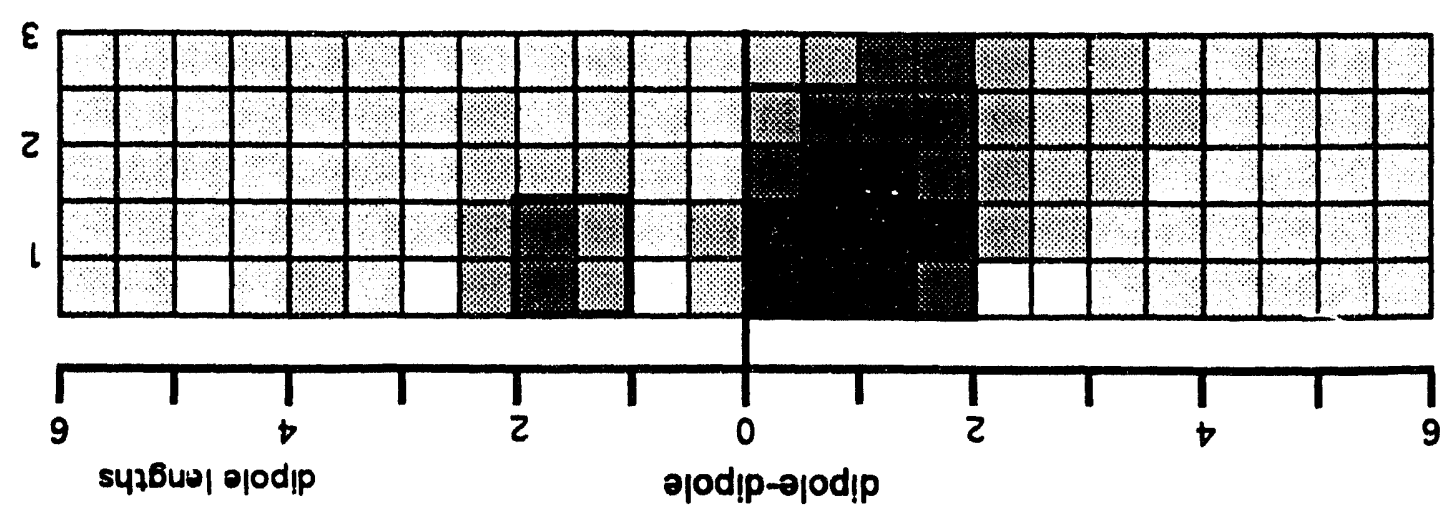

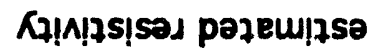

so!poq OM? 
(a)

prism with conductive cover

$$
\text { dipole-dipole }
$$

\section{Resistivity}
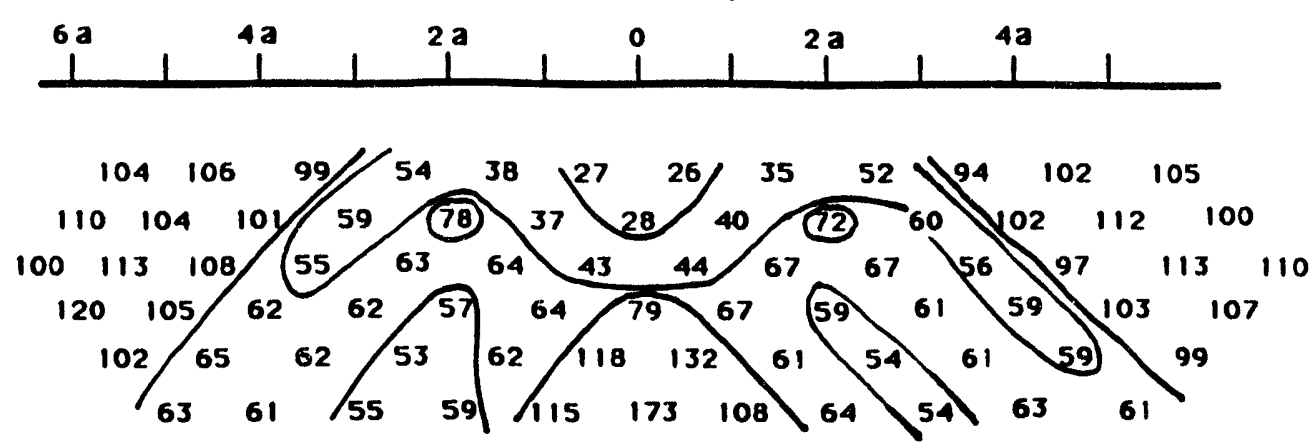

(b)

$$
\text { pole-dipole }
$$

\section{Resistivity}
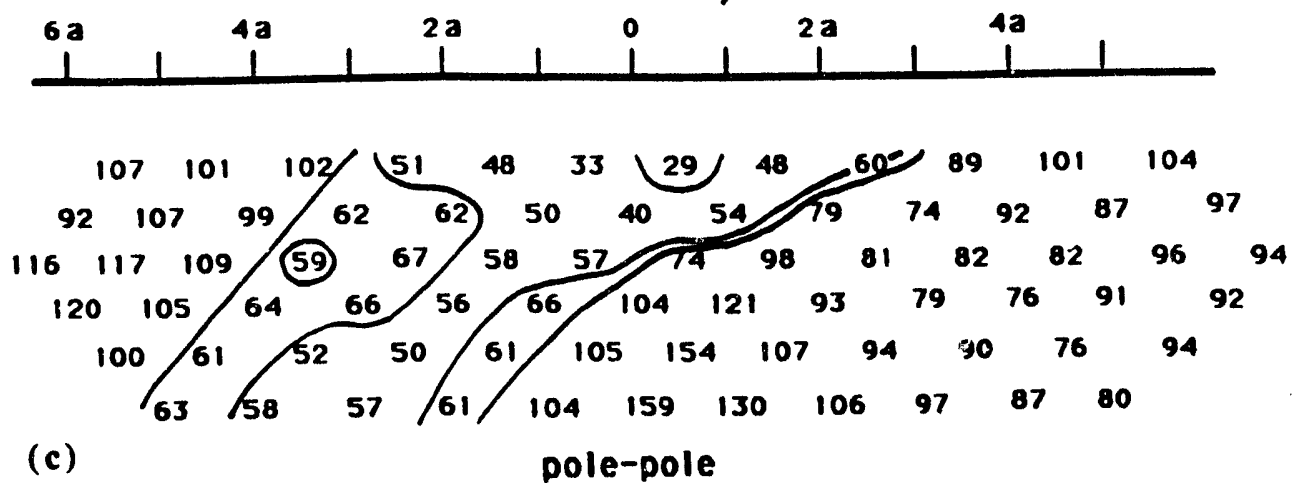

(c)

\section{Resistivity}

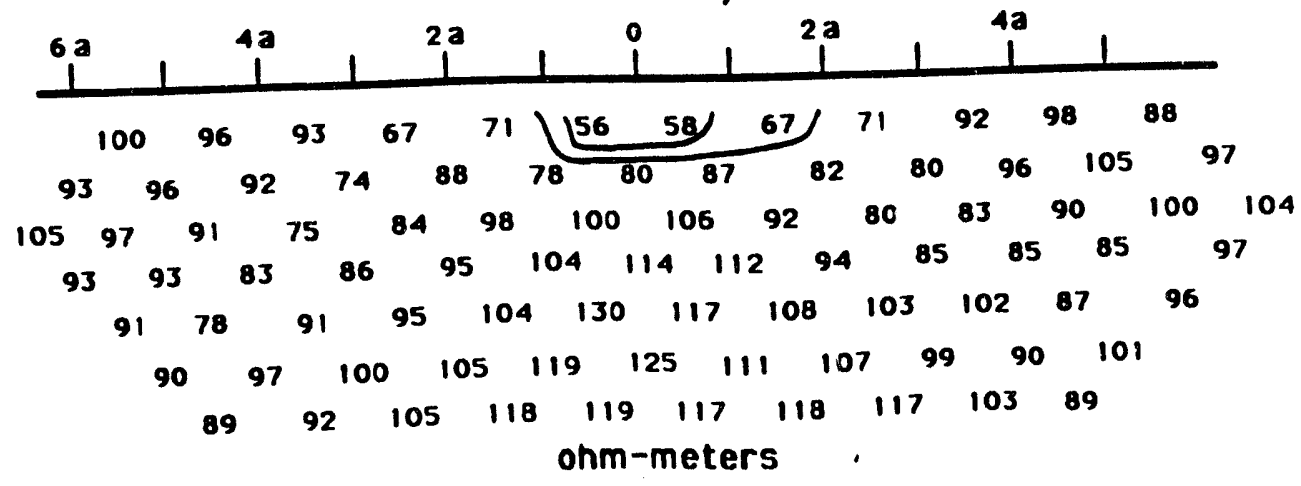

FIG. 5. Apparent resistivity data with 5\% Gaussian noise for different arrays over a conductor masked by conductive overburden. (a) Dipole-dipole pseudosection. (b) Pole-dipole pseudosection. (c) Pole-pole pseudosection. 


\section{prism with conductive cover}

\section{estimated resistivity}

dipole-dipole

(a)

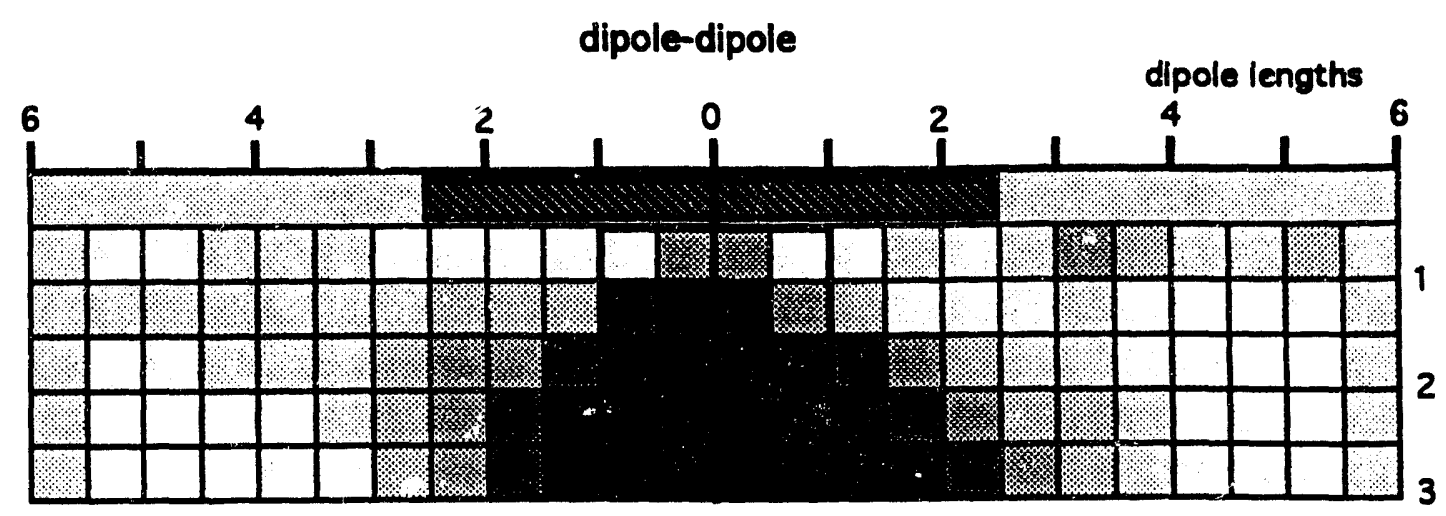

pole-dipole

(b)

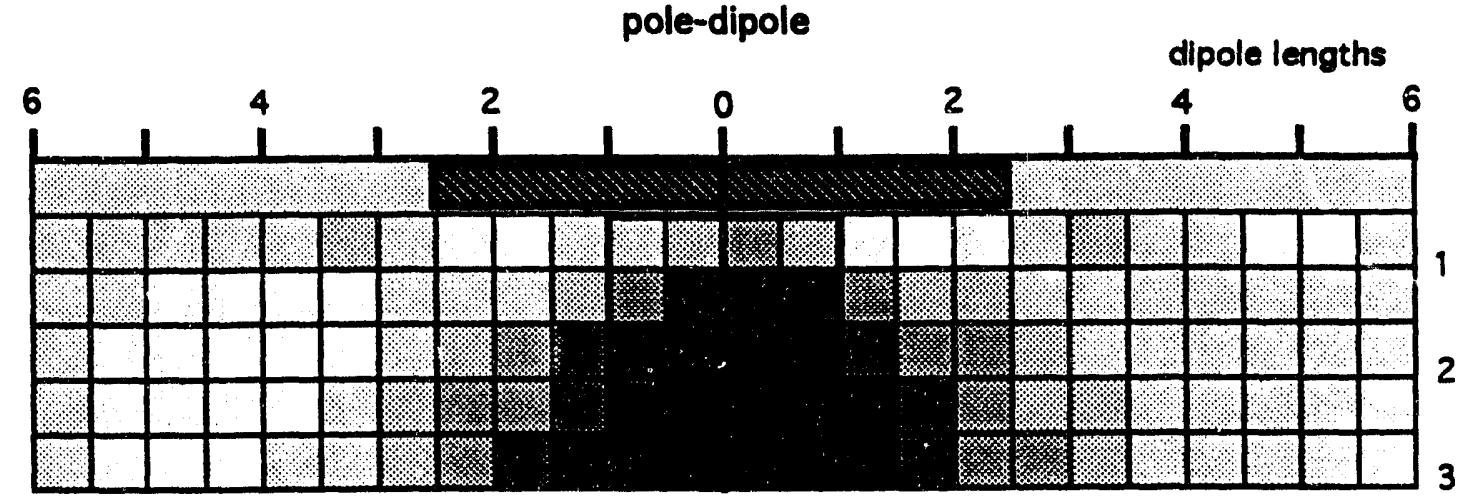

pole-pole
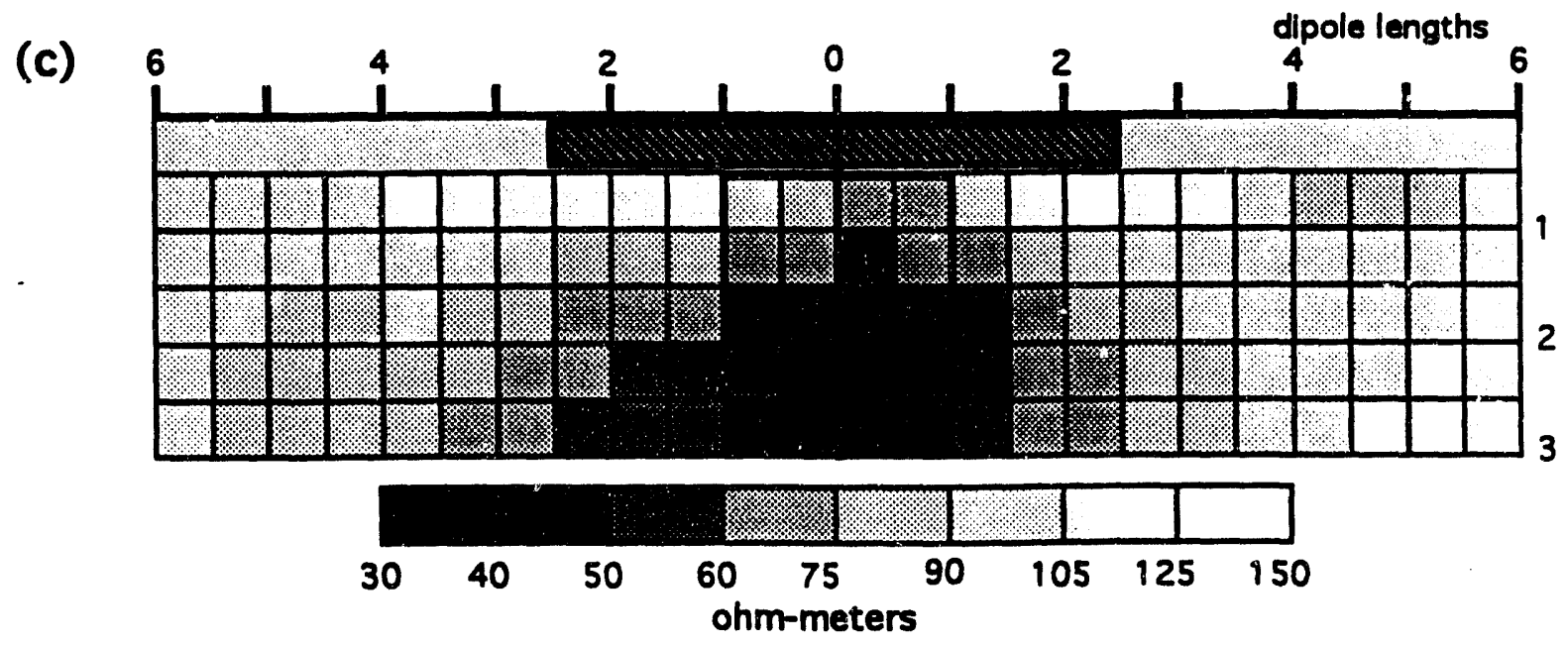

bg $=100 \mathrm{ohm}-\mathrm{m}$

FIG. 6. Final estimated resistivity models from inversion of data over the conductor masked by conductive overburden. (a) Dipole-dipole final estimated model. Resistivity $\chi_{v}{ }^{2}=0.71$. (b) Pole-dipole final estimated model. Resistivity $\chi_{v}{ }^{2}=1.04$. (c) Pole-pole final estimated model. Resistivity $\chi_{v}{ }^{2}=0.97$. 
Apparent resistivity in ohm- $n$

$2 \times 2 \times 2$ dipole, $10 \mathrm{ohm}-\mathrm{m}$ cube

(a)

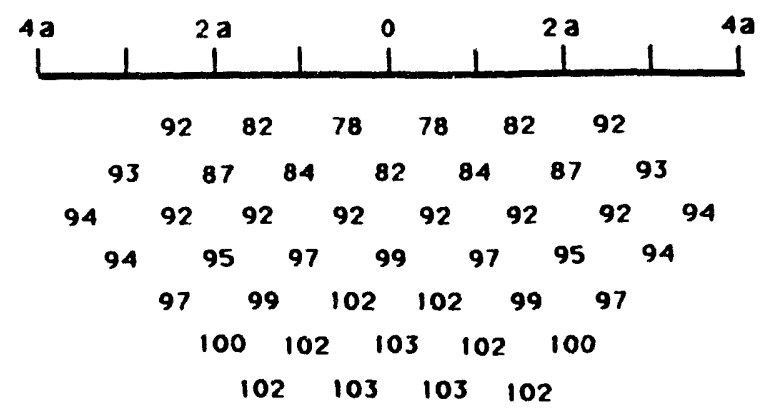

(b)

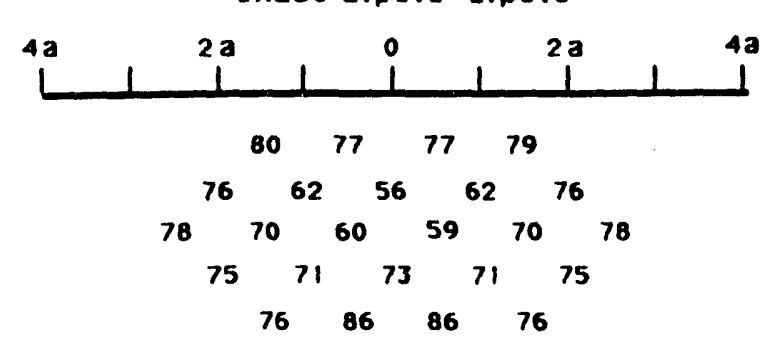

(c)

transformed dipole-dipole, 0.18 noise

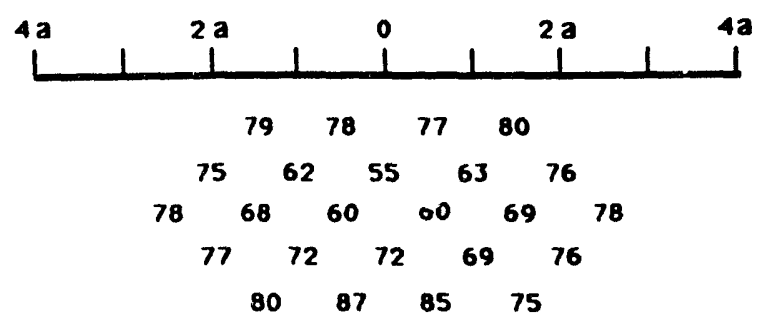
transformed dipole-dipole, 0.47 noise

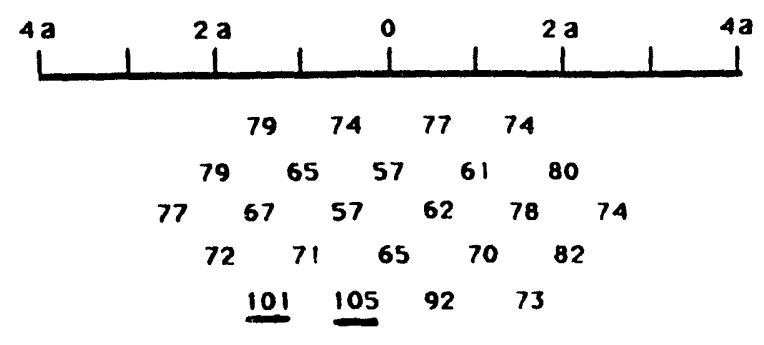

transformed dipole-dipole, $1.0 \%$ noise

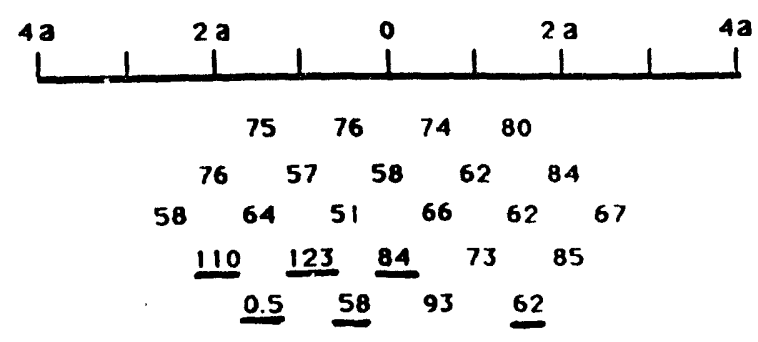

FIG. 7. Effect of noise on transformation of pole-pole apparent resistivity data to dipole-dipole data via superposition. Underlined numbers in $(\dot{d})$ and $(e)$ indicate a difference of more than $15 \%$ with the exact apparent resistivities in (b). (a) Exact pole-pole data computed from $2 \times 2 \times 2$ (dipole length) ${ }^{3}, 10 \Omega-\mathrm{m}$ cube buried 0.5 dipole length deep in $100 \Omega-\mathrm{m}$ earth. (b) Exact dipole-dipole apparent resistivity data computed from same body. (c) Dipole-dipole apparent resistivities from differenced pole-pole data with $0.1 \%$ random noise. (d) Dipole-dipole apparent resistivities from differenced pole-pole data with $0.4 \%$ random noise. (e) Dipoledipole apparent resistivities from differenced pole-pole data with $1.0 \%$ random noise. 

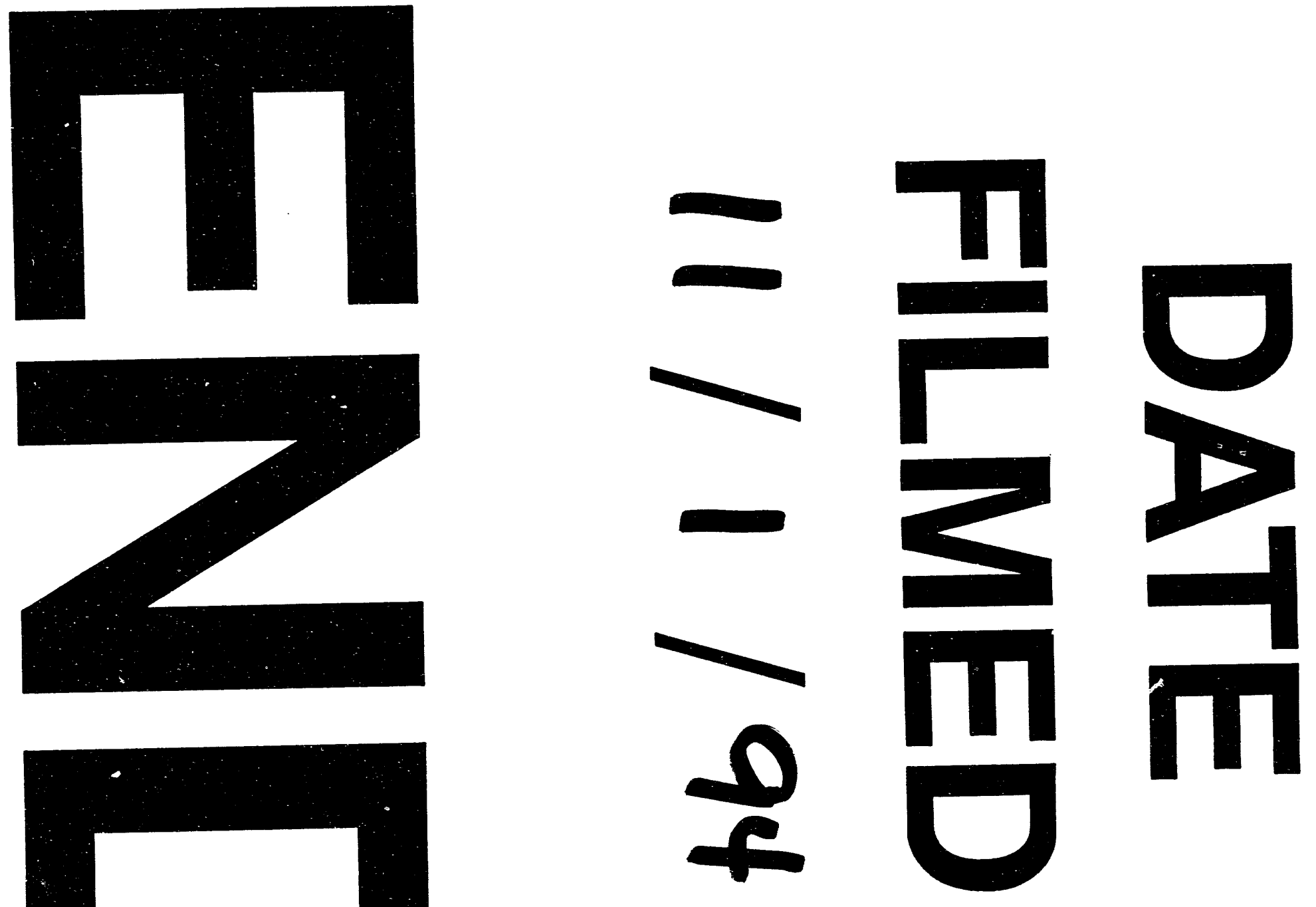
- 